\title{
BMJ Open Constraint choice in revision knee arthroplasty: study protocol of a randomised controlled trial assessing the effect of level of constraint on postoperative outcome
}

\author{
Hagen Hommel, ${ }^{1,2}$ Kai Wilke, ${ }^{1}$ Daniel Kunze, ${ }^{1}$ Peggy Hommel, ${ }^{1}$ Peter Fennema ${ }^{3}$
}

\begin{abstract}
To cite: Hommel $\mathrm{H}$, Wilke $\mathrm{K}$, Kunze $\mathrm{D}$, et al. Constraint choice in revision knee arthroplasty: study protocol of a randomised controlled trial assessing the effect of level of constraint on postoperative outcome. BMJ Open 2017;7:e012964. doi:10.1136/bmjopen-2016012964
\end{abstract}

- Prepublication history for this paper is available online. To view these files please visit the journal online (http://dx.doi.org/10.1136/ bmjopen-2016-012964).

Received 7 June 2016 Revised 4 January 2017 Accepted 14 February 2017

CrossMark

\section{${ }^{1}$ Krankenhaus Märkisch Oderland GmbH BT Wriezen, Klinik für Orthopädie, Sportmedizin und Rehabilitation, Wriezen, Germany \\ ${ }^{2}$ Lehrkrankenhaus der Medizinischen Hochschule Brandenburg (MHB) Theodor Fontane, Neuruppin, Germany ${ }^{3}$ AMR Advanced Medical Research GmbH, Männedorf, Switzerland}

Correspondence to Priv.-Doz. Dr. med. Hagen Hommel; H.Hommel@khmol.de

\section{ABSTRACT}

Introduction: The proper management of total knee arthroplasty (TKA) in patients with severe deformities regarding the preferable prosthetic design and the required amount of constraint is a controversial subject. In the absence of any high-level clinical evidence, we designed a randomised clinical trial to investigate if rotating hinged (RTH) and constrained condylar knee (CCK) designs yield similar outcomes. Methods and analysis: This study is a multicentre, randomised clinical trial including two groups of 85 patients. Patients will be randomised to a CCK knee design group or an RTH knee design group. Patients will be followed for 2 years. The study will be designed as an equivalence trial. The primary study outcome will be the postoperative functional outcome as measured by the self-administered Knee Injury and Osteoarthritis Outcome Score. Secondary outcomes will be postoperative joint awareness during various activities of daily living as measured by the Forgotten Joint Score-12, the Knee Society Score, along with the incidence and location of radiolucent lines using the Knee Society TKA radiographic evaluation system.

Ethics and dissemination: This study is approved by the ethics committee of the Landesärztekammer Brandenburg ((S 10(a)/2013) from 27.08.2013, amended on 25.04.2016) and will be conducted according to the principles of the World Medical Association Declaration of Helsinki and the IS014155:2011.

Trial registration number: DRKS00010539.

\section{INTRODUCTION}

The global increase in the incidence of total knee arthroplasty (TKA) has led to a concurrent rise in the incidence of complex primary and revision arthroplasty (RTKA) procedures. One study estimated the overall incidence of primary TKA and RTKA to grow by $174 \%$ and $600 \%$, respectively, between 2005 and $2030 .^{1}$

\section{Strengths and limitations of this study}

- This is the first randomised clinical study to assess differences in outcome between rotating hinged and condylar constraint knee arthroplasty.

- More insight will be gained into the problem of implant constraint, with patient reported outcome measures focusing on patient's relevancy.

- Limitation is the heterogeneity of the study population in terms of indication for surgery.

One of the major challenges of TKA is the management of instability, a factor which is a prerequisite for postoperative function as well as implant survival. ${ }^{2}$ Overcoming ligamentous instability requires a challenging combination of managing soft tissue deficiency, balancing the flexion and extension gaps and managing extensor mechanism insufficiency. ${ }^{3}$ The choice of implant constraint depends on the state of the ligaments of the knee and the severity of bone loss. ${ }^{2}$ The best pairing between bony defects during RTKA and the level of implant constraint required is a controversial topic. Posterostabilised designs may be appropriate when the ligaments of the knee are intact and bone defects are minimal. ${ }^{2} 4$ Hinged knee prostheses with a fixed axis were introduced to restore knee function and correct limb alignment in the presence of severe malalignment and/or instability. ${ }^{5}$ Disappointing long-term outcomes with low survival rates prompted a further refinement of the design of these prostheses. ${ }^{6}$ The rotating hinged (RTH) total knee prosthesis was introduced in $1982,{ }^{7}$ and is typically used in cases of ligament absence or ligament disruption in combination with moderate or severe bone 
loss. ${ }^{2}$ A potential advantage of this knee design is the reduction of shear stresses around the bone-cement interface due to the additional rotation around the tibial axis. ${ }^{9} 10$

Studies reporting outcome of different types of RTH implants have been published; some had controversial conclusions. ${ }^{811-15}$

Condylar constrained knee (CCK) prostheses are uncoupled, non-hinged and semiconstrained implants. In patients where gross joint instability is not an issue, CCK prostheses represent an alternative to the RTH prostheses. CCK systems have been introduced more recently, and their use has become increasingly popular in patients with intermediate-but not complete-insufficiency of the ligaments and moderate bone loss. ${ }^{2}$

The preferable prosthetic design and required amount of constraint is still a controversial area. ${ }^{16}{ }^{17}$ A significant shortcoming of constraint in TKA stems from the major increase of force transfer to the bone-implant interface. CCK prostheses are generally believed to be less constrained than RTH designs. CCK designs provide mediolateral and rotational constraint but no anteroposterior constraint. It should be taken into account that RTH prostheses offer more freedom in the axial plane than CCK designs. ${ }^{3}$ Previous studies have reported that RTH prostheses may, in contrast to common beliefs, be less constrained than CCK designs. Finite element models have shown that RTH designs are prone to less shear stress at the bone-implant interface when compared with CCK designs. ${ }^{18}$ However, there is a paucity of studies focussing on the clinical relevance of this difference in shear stress. Observational studies have shown that when implant selection is guided by inherent stability of the knee, CCK and RTH knee designs have similar outcomes. ${ }^{3}$ Fuchs et $a l^{19}$ found no significant differences in terms of Hospital for Special Surgery Score, Knee Society Score (KSS), Pain and the Tegner Score in their retrospective cohort study. Standard condylar revision implants yielded better postoperative mobility but had a lower Short Form 36 Mental Component Score. The latter finding may imply that patients tolerate the RTH design better than the standard condylar revision implant. Walker et $a l^{20}$ also compared the RTH design with standard condylar revision in a cohort study and found a high correlation in performance between the operated and non-operated side in the RTH group, indicating the hinges were capable of matching the non-operated knee performance.

In our clinic, we studied 74 consecutive patients receiving a CCK design during primary or revision TKA from 2007 to $2013 .^{21}$ This patient group was compared with a historical cohort of patients $(n=93)$ receiving an RTH prosthesis as a primary or revision implant from 2003 to 2007. Table 1 summarises the baseline characteristics of the patient populations.

The differences between follow-up and baseline values between the cohorts were compared (difference in difference (DID)). DIDs between CCK and RTH arthroplasty did not deviate significantly for either of the subscores of
Table 1 Baseline characteristics of the study population ${ }^{21}$

\begin{tabular}{|c|c|c|c|}
\hline & CCK & RTH & p Value \\
\hline Females:males* & $42: 32$ & 69:33 & $0.307 \dagger$ \\
\hline Age (years) $\ddagger$ & $69.8 \pm 9.0$ & $72.1 \pm 4.3$ & $0.042 \S$ \\
\hline BMI $\left(\mathrm{m} / \mathrm{kg}^{2}\right) \ddagger$ & $30.0 \pm 3.1$ & $31.1 \pm 3.4$ & $0.028 \S$ \\
\hline $\mathrm{ASA}^{*}$ & $18 / 14 / 12$ & $14 / 57 / 22$ & $0.224 \dagger$ \\
\hline HKA (varus/valgus)* & $55: 19$ & $66: 27$ & $0.630 \dagger$ \\
\hline \multicolumn{4}{|l|}{ Baseline scores } \\
\hline KS $\ddagger$ & $24 \pm 4$ & $26 \pm 4$ & $0.031 \S$ \\
\hline FS $\ddagger$ & $20 \pm 5$ & $22 \pm 6$ & $0.001 \S$ \\
\hline WOMAC $\ddagger$ & $65 \pm 6$ & $65 \pm 6$ & $0.741 \S$ \\
\hline
\end{tabular}

${ }^{*}$ Presented as number of observations.

†Pearson's chi-squared test.

$\ddagger$ Presented as mean \pm SD.

$\S S t u d e n t ' s$ t-test.

ASA, American Society of Anesthesiologists Score; BMI, body mass index; CCK, constrained condylar knee; FS, Function Score $\mathrm{KS}$, Knee Score; RTH, rotating hinged; WOMAC, Western Ontario and McMaster Universities Osteoarthritis Index.

the KSS (the Function Score (FS) and the Knee Score (KS)) or for the Western Ontario and McMaster Universities Arthritis Index (WOMAC). Subgroup analysis of patients with valgus and varus osteoarthritis at the primary intervention yielded clinically equivalent results for the varus group, but significantly better KSSs were obtained in patients with an initial valgus deformity who had received an RTH prosthesis (table 2).

None of the studies were randomised, and confounding-by-indication is likely to have biased the conclusions of the aforementioned trials. In the absence of any high-level clinical evidence, we therefore designed a randomised clinical trial to investigate if RTH and CCK designs yield similar outcomes.

\section{METHODS AND ANALYSIS}

Based on the findings from observational studies, we designed the present randomised trial to verify the impact of implant constraint on the outcome of revision

Table 2 'Difference in difference' in clinical outcome between CCK and $\mathrm{RTH}^{21}$

\begin{tabular}{lccl}
\hline & CCK & RTH & p Value \\
\hline Follow-up time (months) & $54 \pm 25$ & $106 \pm 33$ & $<0.001$ \\
KS & $63 \pm 6$ & $63 \pm 4$ & 0.712 \\
$\quad$ Varus & $65 \pm 5$ & $63 \pm 4$ & 0.039 \\
$\quad$ Valgus & $57 \pm 4$ & $63 \pm 5$ & $<0.001$ \\
FS & $64 \pm 8$ & $64 \pm 7$ & 0.995 \\
$\quad$ Varus & $66 \pm 8$ & $64 \pm 7$ & 0.082 \\
$\quad$ Valgus & $59 \pm 6$ & $65 \pm 8$ & 0.025 \\
WOMAC & $-40 \pm 7$ & $-39 \pm 8$ & 0.510 \\
$\quad$ Varus & $-41 \pm 7$ & $-39 \pm 7$ & 0.177 \\
$\quad$ Valgus & $-37 \pm 7$ & $-39 \pm 8$ & 0.351 \\
\hline
\end{tabular}

Presented as mean \pm SD.

$\S$, Student's t-test.

CCK, constrained condylar knee; FS, Function Score; KS, Knee Score; RTH, rotating hinged; WOMAC, Western Ontario and McMaster Universities Osteoarthritis Index. 
TKA. With this study, we plan to assess if revision knee arthroplasty using an RTH knee design and a standard condylar knee design have equivalent functional outcomes.

Secondary objectives are to demonstrate that, compared with a CCK design, RTH knee design leads to similar clinical and radiographic outcomes, and has a similar complication rate. In addition, the ability to forget the knee prosthesis during activities of daily life will be compared between the two populations.

This study is a multicentre, randomised clinical trial. Patients will be randomised before the surgical procedure to receive a CCK knee design (Legion, Smith and Nephew GmbH, Hamburg, Germany) or an RTH knee design (RT-Plus Solution, Smith and Nephew GmbH). Study participants will be allocated to one of the two study arms in a 1:1 ratio. Patients will be followed for two years after inclusion. This study was designed as an investigator-initiated trial, and the institution of the principal investigator $(\mathrm{HH})$ will take the role of the sponsor. The study will be performed at four community clinics in Germany. Only clinics that perform at least 50 total knee revisions per year will be eligible.

The current study will be designed as an equivalence trial: the study was designed to show that the two interventions do not differ in either direction by more than a prespecified unimportant or insignificant amount. ${ }^{22}$

All medical devices are used in the routine manner as specified in each product's instructions for use. Only devices with prior market authorisation will be applied in the study.

The study protocol was drafted following the SPIRIT statement ${ }^{23}$ and the CONSORT extension for reporting noninferiority and equivalence trials. ${ }^{24}$

\section{Outcomes}

The following characteristics will be retrieved from patient questionnaires, physical examination, the hospital information system or medical records: patient characteristics (ie, sex, age, height and weight); indication for surgery; American Society of Anesthesiologists classification; previous joint procedures or injury; present degree of deformity and degree of deformity before the index procedure; comorbidities; smoking and alcohol consumption and pain medication consumption.

We will also note arthroplasty-related characteristics such as anaesthesia method, type of arthroplasty, surgical approach, postoperative analgesic consumption and arthroplasty-related complications.

The primary outcome of the study will be the postoperative functional outcome as measured by the selfadministered Knee Injury and Osteoarthritis Outcome Score (KOOS). ${ }^{25} 26$ The KOOS was developed in the 1990s to assess patients' opinions on the state of their knees after injury or surgery. ${ }^{26}{ }^{27}$ The score also has sufficient sensitivity in the younger and more active patients with knee problems. In addition to questions about pain and stiffness, it examines knee function in daily life, sport and recreational activities and the quality of life.

Secondary outcomes of this study are:

- Joint awareness during various activities of daily living, as measured by the Forgotten Joint Score-12 (FJS-12) ${ }^{28}$ The FJS-12 is a recently published patientreported outcome measure to assess joint awareness in hips and knees during various activities of daily living. ${ }^{28}$ It consists of 12 questions. ${ }^{29}$ The FJS-12 has a high internal consistency (Cronbach's $\alpha$ 0.95), a low ceiling effect and the instrument discriminates well between good, very good and excellent outcomes after total hip arthroplasty (THA) and TKA. ${ }^{29}$

- Patients' knees and functional abilities such as walking and stair climbing before and after TKA as assessed via the KSS, a simple, objective, globally recognised scoring system ${ }^{30}$ to track and report outcomes after total and partial knee arthroplasty.

- Conventional anteroposterior and lateral radiographs will be taken at designated time points. Postoperative radiographs will assess component position, alignment and the location and incidence of radiolucent lines using the Knee Society TKA radiographic evaluation system. ${ }^{31}$

- The incidence of adverse events (AEs) and adverse device effects.

\section{Study population}

For a patient to qualify for study enrolment, the CCK and the RTH design should be indicated. If one of the two designs prevails in the physician's reasonable medical judgement, the patient should receive the treatment deemed most suitable by the patient's physician.

Study participants will be recruited from eligible patients at the orthopaedic department of the Krankenhaus Märkisch Oderland GmbH BT, Wriezen, Germany scheduled for knee arthroplasty, and from three other clinical sites that have yet to be recruited. Eligible patients must require primary or revision surgery and must have an axial malalignment $>18^{\circ}$, with one of the collateral ligaments (ie, medial collateral and/or lateral collateral ligament) being absent or incompetent. $^{15}$

Indications are: ${ }^{32}$

- rheumatoid arthritis;

- post-traumatic arthritis, osteoarthritis or degenerative arthritis in older patients whose age, weight and activity levels are compatible with an adequate long-term result;

- failed osteotomies, unicompartmental replacement or total knee replacement.

In addition, the patient has to fulfil the following criteria to be eligible for the study:

- The patient is scheduled for elective primary or revision knee arthroplasty.

- The patient is 18 years or older. There are no upper age limits for the study, as long as the patient is healthy enough to undergo surgery. 
- The patient is willing to provide informed consent.

The following exclusion criteria shall be used for the study:

- genu recurvatum;

- gross anteroposterior instability;

- the patient participates in another concurrent study;

- major psychiatric disease;

- patients who do not comprehend the German language (read and speak);

- pregnancy, lactation or childbearing potential without using adequate contraception;

- acute or chronic infections, local or systemic (or corresponding previous incidents);

- severe muscle, nerve or vascular diseases that endanger the extremity in question;

- lacking bone substance or possessing inadequate bone quality that endangers a stable seating of the prosthesis;

- extreme insufficiency of the knee extensor mechanism as this can lead to excessive joint distortion;

- adipositas permagna;

- local tumours;

- known hypersensitivity to the material.

\section{Devices}

The Legion Revision System (Smith and Nephew, Memphis, Tennessee, USA) was introduced in Europe in 2006, and good postoperative outcome has been reported with the system. ${ }^{33}$ The system is available with a variety of wedges and femoral and tibial stems with and without offset. It is indicated for use in total knee replacement to overcome severe ligamentous imbalance, laxity or in the presence of deformity in excess of $20^{\circ}$.

The RTH RT-Plus (Smith and Nephew) was introduced in Europe in 1997, and we previously reported good mid-term to long-term outcome with this device. ${ }^{15}$ The system is available with a variety of wedges and femoral and tibial stems. For the study, the device will be used within its indications for use while excluding severe ligamentous imbalances, ligamentous laxities or gross deformities in excess of $20^{\circ}$.

\section{Study withdrawal}

In accordance with the Declaration of Helsinki and with local regulations, study subjects have the right to withdraw at any time from the study without providing a reason. The investigator or regulatory authority can discontinue a subject's participation in the study at any time if medically or otherwise necessary. Unless the subject wishes to withdraw from the study completely, all scheduled examinations will be performed as planned.

\section{Study hypothesis}

The current study will be designed as an equivalence trial, that is, a trial designed to show that the two interventions do not differ in either direction by more than a prespecified unimportant or insignificant amount. ${ }^{34}$ The amount of permissible difference is the margin that delineates the 'zone of indifference'. Within this zone of indifference, the two groups are considered equivalent. $^{35}$

The KOOS has a minimally clinically important difference (MCID) of 8 to 10 points, and the SD in a cohort of TKA patients is $\sim 15$ points. ${ }^{26}$

The null hypothesis (of non-equivalence) of the study is that functional outcome at 2 years of follow-up in the $\mathrm{RTH}$ group is not equal to the functional outcome at 2 years of follow-up in the CCK group, or:

$$
\mathrm{H}_{0}: \vartheta \leq \vartheta_{0}-\delta_{1} \text { or } \vartheta \geq \vartheta_{0}+\delta_{1}
$$

versus the equivalence assumption that functional outcome at 2 years of follow-up in the RTH group is equivalent to the functional outcome at 2 years of follow-up in the CCK group, or:

$$
\mathrm{H}_{0}: \vartheta_{0}-\delta_{1}<\vartheta<\vartheta_{0}+\delta_{1} .
$$

\section{Sample size calculation}

With $\alpha=0.05$ and $\beta=0.10$, a sample size between 49 $(\mathrm{MCID}=10)$ and $77 \quad(\mathrm{MCID}=8)$ will be required. Calculation is based on the following formula: ${ }^{36}$

$$
\mathrm{n}=\mathrm{f}(\alpha, \beta / 2) \times 2 \times \alpha^{2} \times \delta^{2} / 2
$$

with:

$$
\mathrm{f}(\alpha, \beta)=\left[\Phi^{-1}(\alpha)+\left[\Phi^{-1}(\beta)\right]^{2} .\right.
$$

Attrition and dropout rate at the 2-year follow-up is estimated to be $10 \%$. To account for attrition and drop-out, a total of $2 \times 85$ patients will be enrolled.

\section{Randomisation}

Patients will be randomly allocated to the treatment or control group in a 1:1 ratio, using block randomisation with random block sizes. Stratification based on study site will be performed. Under all circumstances, subjects will not be randomised before they have provided informed consent. Randomisation in advance of surgery (by at least 24 hours) is required in order to allow proper preoperative planning of the surgery, alongside preparation of the instrument sets. The investigator will randomise the patient through the internet (http:// www.randomisation.com) and document the treatment group in the patient's chart.

\section{Blinding}

Surgeon and patients will not be blinded for the assigned treatment. In order to preserve the blinding of the study, a minimum number of examiners will have access to the randomisation schedule before the study is complete. To reduce measurement bias, the preoperative and follow-up assessments will be performed by independent examiners blinded to group allocation (one examiner plus one back up at each centre, both 
trained and neither involved in surgery). Before each follow-up visit, subjects will be reminded not to disclose their allocation.

One independent single examiner will perform the radiographic assessments. Blinding of this examiner will not be possible.

One interim analysis is planned to investigate the safety and the primary outcome after half of the patients have been enrolled. The statistician will provide a summary of the AEs and an analysis of the primary outcome. Study groups will be blinded; the group assignments will not be revealed. These results will be presented to the Clinical Study Steering Committee (CSSC).

Based on the interim analysis, the CSSC will then decide whether to continue the study without adjustment, continue the study with adjustment(s) or to stop the study due to safety or efficacy concerns. The CSSC may request to reveal study arm assignments before coming to a definitive decision. Early termination of the study for efficacy at the interim analysis will be considered if the benefit of either one of the study groups is shown 'beyond reasonable doubt.' A nominal $\mathrm{p}$ value $<0.003$ (O'Brien Fleming $\alpha$ spending ${ }^{37}$ ) provides guidance in this instance but is not binding for this decision. As the products used in both study arms are CE-marked and are present in the market for $<10$ years, no formal criteria for early stopping for safety reasons have been defined. The CSSC's decision will be based on the statistical analysis and the clinical expertise of the board members.

The interim results will be confidential and strictly limited to the members of the CSSC. This confidentiality minimises the risk of interim-result bias on the remaining course of the trial.

All analyses will be performed primarily as an intention-to-treat-analysis, assessing all patients with available data for the outcomes according to the randomisation. For baseline characteristics, descriptive statistics will be used as appropriate. The primary hypothesis will be tested using the Westlake version of an equivalence test with known and fixed delta. ${ }^{38}$ Further exploration of the primary and secondary outcomes will be based on linear mixed models. ${ }^{39}$ Inferences on the random effect structures will be based on the restricted maximum likelihood and inferences for the fixed effect structure will be based on the standard maximum likelihood.

With the exception of the interim analysis of the primary outcome, two-sided significance tests with an $\alpha$ of 0.05 will be applied. Stata 12.1 (StataCorp, College Station, Texas, USA) will be used for the analysis.

\section{Study procedures}

Patients that are screened and deemed ineligible prior to study inclusion will be tracked on a screening log along with a detailed reason for exclusion.

Patients who are deemed eligible but who are not included in the study will be tracked on a screening log along with the reason for exclusion.
Patients that are enrolled and are deemed ineligible during surgery will be tracked on a dedicated Termination Case Report Form along with the reason for exclusion.

\section{Informed consent}

Patients will be informed verbally by the investigator about the study objectives, design, risks, the study procedures and patient rights. Only patients who have signed the informed consent form 24 hours before surgery and meet all inclusion criteria and no exclusion criteria will be included in the trial. Patients will receive a copy of the signed informed consent form.

\section{Study visit definitions}

The screening date is defined as the date the informed consent is signed. The enrolment date is defined as the date of randomisation. Day 1 is defined as the surgery day. All on-study visits will be calculated from the initial day-1 visit. However, if a subject's visit is delayed, the subsequent visit date will be shifted.

Study visit windows will be \pm 7 days for all the visits of the treatment and observation period and \pm 14 days for follow-up visits at the 1-year and 2-year follow-ups. Study procedures for a specific visit may be completed on multiple days as long as all the procedures are completed within the visit window. The subject's medical history will be obtained prior to enrolment and recorded in an electronic data capturing system. Table 3 shows all the assessments and procedures performed during each visit.

\section{Concomitant care}

It is imperative that all study participants receive the best available care. This may lead to differences in concomitant care between the study groups. All concomitant medications and treatments will therefore be reported on a concomitant care case report form.

\section{Dissemination}

This clinical study will be conducted in accordance with the latest version of the World Medical Association Declaration of Helsinki, ISO14155, and German regulations. The study is registered at the German Clinical Trials Register (trial registration number DRKS00010539). The ethics committee will be notified should future amendments (ie, modifications that are likely to affect the safety or the scientific value of the trial) arise.

The results of the study will be published in international peer-reviewed scientific journals, independent from the outcome. Anonymous patient data will be made available on request of the journal. We will provide a copy of the protocol, including the statistical analysis plan, to the medical journal considering a submitted manuscript for publication, if requested by the journal. 
Table 3 Schematic timeline

\begin{tabular}{|c|c|c|c|c|c|c|c|c|}
\hline \multirow[b]{3}{*}{ Time point } & \multicolumn{8}{|c|}{ Study period } \\
\hline & \multirow{2}{*}{ Enrolment } & \multirow{2}{*}{ Allocation } & \multicolumn{5}{|c|}{ Postallocation } & \multirow{2}{*}{$\begin{array}{l}\text { Close-out } \\
2 \text { years }\end{array}$} \\
\hline & & & Baseline & Peri-op & 3 months & 6 months & 1 year & \\
\hline \multicolumn{9}{|l|}{ Enrolment: } \\
\hline Eligibility screen & $x$ & & & & & & & \\
\hline Informed consent & $x$ & & & & & & & \\
\hline Allocation & & $x$ & & & & & & \\
\hline Surgery & & & & $\mathrm{X}$ & & & & \\
\hline \multicolumn{9}{|l|}{ Assessments: } \\
\hline PT characteristics & & & $\mathrm{X}$ & & & & & \\
\hline KOOS & & & $x$ & & $x$ & $x$ & $x$ & $x$ \\
\hline KSS & & & $\mathrm{X}$ & & $\mathrm{X}$ & $x$ & $x$ & $x$ \\
\hline FJS & & & & & $x$ & $x$ & $x$ & $x$ \\
\hline Radiography & & & & & $x$ & $x$ & $\mathrm{x}$ & $\mathrm{x}$ \\
\hline Adverse events & & & & $x$ & $x$ & $x$ & $x$ & $x$ \\
\hline
\end{tabular}

\section{Authorship}

The International Committee of Medical Journal Editors (ICMJE) recommendations regarding authorship will be followed. The investigators will identify the individuals who accept direct responsibility for the publication. All authors must take responsibility for the manuscript. Publications from individual institutions participating in the study will not precede the primary manuscript. An external professional medical or scientific writer may be employed to assist in producing publications. Names, involvement and funding of the external writer will be acknowledged in the publication. We will not engage ghostwriters.

\section{Study organisation}

No data and safety monitoring board will be installed, as the safety and efficacy of the study devices have been documented. A CSSC has been installed consisting of the principal investigators of the four study sites and a statistician. The CSSC has the primary responsibility for designing the study, maintaining the quality of study conduct, the ongoing monitoring of safety and efficacy and writing the study publications. The committee will also assess whether the endpoints meet the criteria of equivalence. Except for the statistician, the committee will be masked to the assigned study arm when performing their assessments.

\section{Data collection and monitoring}

Patients will be hospitalised during the whole study (due to the RTKA procedure). Study participants will therefore be under steady monitoring by surgeons and regular hospital staff. Dedicated study physicians and study nurses will collect data from the patients' records. Study nurses will schedule patient follow-ups to the clinic and will oversee the physical and the radiographic assessments. Study nurses will also provide and collect the questionnaires from the patients and will provide assistance with completing the questionnaires if requested by the patient. The clinical data management system used for the study will be the 21 CFR Part 11 and GCP compliant, CDISC-certified electronic data capture system Marvin (XClinical GmbH, Munich, Germany). Patients will be coded and patient-identifiable information will not be stored in the clinical data management system.

Data entry comprises extensive data edit checks. The software will dynamically check and query illegal ranges and inconsistent entries. Data entry is recorded in an audit trail, and backup to a remote server is performed several times per day.

The sponsor has appointed an independent contract research organisation (CRO) to perform site/clinical monitoring of the study to assure high-quality trial conduct. A monitor appointed by the CRO will perform biannual 'on site' monitoring of individual case histories, assess adherence to the protocol, ensure the ongoing implementation of appropriate data entry and quality control procedures and, in general, assess adherence to good clinical practices. ${ }^{40}$ The study monitor will remain blinded to study arm assignment.

\section{AEs and adverse device effects}

All AEs and adverse device effects reported spontaneously by the study subject or observed by the investigators or staff will be recorded. In case of a serious adverse device effect (SADE), the principal investigator will report the SADE to the ethics committee and the authorities (Bundesinstitut für Arzneimittel und Medizinprodukte (BfArM)). Prolonged hospitalisation (>14 days) will be reported as an SAE or as an SADE. Rehospitalisation (for any reason) will also be reported and handled as an SAE or as an SADE. All AEs will be followed until the event has resolved, or until a stable situation has been achieved. 


\section{STUDY LIMITATIONS}

There are several potential limitations to this study. First, heterogeneity of the study population in terms of indication for surgery introduces randomness in the data. The study is likely to be underpowered to assess possible effect modifiers such as preoperative deformity. Second, having a blinded examiner renders study procedures considerably more complex: the blinded examiner and the study nurse must have no contact with the surgical team; subjects must be briefed about the importance of keeping the examiner blinded.

\section{Twitter Follow Peter Fennema @AMR_CR0}

Contributors $\mathrm{HH}$ and PF participated in the conception of the idea for the study. HH, KW, DK, PH and PF participated in the design of the study and research protocol. $\mathrm{HH}$ and $\mathrm{PF}$ participated in the writing of the manuscript. $\mathrm{HH}, \mathrm{KW}, \mathrm{DK}, \mathrm{PH}$ and $\mathrm{PF}$ were involved in the review and editing of the manuscript. $\mathrm{HH}, \mathrm{KW}, \mathrm{DK}, \mathrm{PH}$ and $\mathrm{PF}$ have critically read the final draft of the paper and approved the final text.

Competing interests $\mathrm{HH}$ is a consultant for Smith and Nephew GmbH, Hamburg, Germany.

Ethics approval Landesärztekammer Brandenburg. (S 10(a)/2013).

Provenance and peer review Not commissioned; externally peer reviewed.

Data sharing statement Anonymous patient data will be made available on request.

Open Access This is an Open Access article distributed in accordance with the Creative Commons Attribution Non Commercial (CC BY-NC 4.0) license, which permits others to distribute, remix, adapt, build upon this work noncommercially, and license their derivative works on different terms, provided the original work is properly cited and the use is non-commercial. See: http:// creativecommons.org/licenses/by-nc/4.0/

\section{REFERENCES}

1. Kurtz S, Ong K, Lau E, et al. Projections of primary and revision hip and knee arthroplasty in the United States from 2005 to 2030. J Bone Joint Surg Am 2007;89:780-5.

2. Vasso M, Beaufils $P$, Schiavone Panni A. Constraint choice in revision knee arthroplasty. Int Orthop 2013;37:1279-84.

3. Malcolm TL, Bederman SS, Schwarzkopf R. Outcomes of varus valgus constrained versus rotating-hinge implants in total knee arthroplasty. Orthopedics 2016;39:e140-8.

4. Meijer MF, Reininga IH, Boerboom AL, et al. Poorer survival after a primary implant during revision total knee arthroplasty. Int Orthop 2013;37:415-9.

5. Lettin AW, Deliss LJ, Blackburne JS, et al. The Stanmore hinged knee arthroplasty. J Bone Joint Surg Br 1978;60-b:327-32

6. Deburge A, Guepar. Guepar hinge prosthesis: complications and results with two years' follow-up. Clin Orthop Relat Res 1976;120:47-53.

7. Walker PS, Emerson R, Potter T, et al. The kinematic rotating hinge: biomechanics and clinical application. Orthop Clin North Am 1982;13:187-99.

8. Hwang SC, Kong JY, Nam DC, et al. Revision total knee arthroplasty with a cemented posterior stabilized, condylar constrained or fully constrained prosthesis: a minimum 2-year follow-up analysis. Clin Orthop Surg 2010;2:112-20.

9. Barrack RL, Lyons TR, Ingraham RQ, et al. The use of a modular rotating hinge component in salvage revision total knee arthroplasty. J Arthroplasty 2000;15:858-66.

10. Neumann DR, Hofstaedter T, Dorn U. Follow-up of a modular rotating hinge knee system in salvage revision total knee arthroplasty. J Arthroplasty 2012;27:814-19.

11. Gehrke T, Kendoff D, Haasper $C$. The role of hinges in primary total knee replacement. Bone Joint J 2014;96-B:93-5.

12. Gudnason A, Milbrink J, Hailer NP. Implant survival and outcome after rotating-hinge total knee revision arthroplasty: a minimum 6-year follow-up. Arch Orthop Trauma Surg 2011;131:1601-7.
13. Joshi N, Navarro-Quilis A. Is there a place for rotating-hinge arthroplasty in knee revision surgery for aseptic loosening? $J$ Arthroplasty 2008;23:1204-11.

14. Hossain F, Patel S, Haddad FS. Midterm assessment of causes and results of revision total knee arthroplasty. Clin Orthop Relat Res 2010;468:1221-8

15. Wilke K, Kunze D, Hommel P, et al. Rotating hinge total knee arthroplasty RT-PLUS Solution: a clinical and radiographic follow-up. J Orthopedics Rheumatol 2016;3:4.

16. Pour AE, Parvizi J, Slenker N, et al. Rotating hinged total knee replacement: use with caution. J Bone Joint Surg Am 2007;89:1735-41.

17. Lombardi AV Jr, Berend KR, Leith JR, et al. Posterior-stabilized constrained total knee arthroplasty for complex primary cases. J Bone Joint Surg Am 2007;89(Suppl 3):90-102.

18. Samiezadeh S, Abolghasemian M, D'Lima DD, et al. Rotating hinge versus constrained condylar knee replacement: which one is more constrained? A finite element study. American Academy of Orthopaedic Surgeons Annual Meeting. New Orleans, LA, 2014.

19. Fuchs S, Sandmann C, Gerdemann G, et al. Quality of life and clinical outcome in salvage revision total knee replacement: hinged vs total condylar design. Knee Surg Sports Traumatol Arthrosc 2004;12:140-3.

20. Walker PS, Manktelow AR. Comparison between a constrained condylar and a rotating hinge in revision knee surgery. knee 2001;8:269-79.

21. Hommel H. Clinical outcome in total knee replacement: hinged vs total condylar design. Wriezen, Germany: Krankenhaus Märkisch Oderland, 2016

22. Greene CJ, Morland LA, Durkalski VL, et al. Noninferiority and equivalence designs: issues and implications for mental health research. J Trauma Stress 2008;21:433-9.

23. Agha RA, Altman DG, Rosin D. The SPIRIT 2013 statement-defining standard protocol items for trials. Int J Surg 2015;13:288-91.

24. Piaggio G, Elbourne DR, Pocock SJ, et al. Reporting of noninferiority and equivalence randomized trials: extension of the CONSORT 2010 statement. JAMA 2012;308:2594-604.

25. Roos EM, Roos HP, Ekdahl C, et al Knee injury and Osteoarthritis Outcome Score (KOOS)--validation of a Swedish version. Scand J Med Sci Sports 1998;8:439-48.

26. Roos EM, Toksvig-Larsen S. Knee injury and Osteoarthritis Outcome Score (KOOS)—validation and comparison to the WOMAC in total knee replacement. Health Qual Life Outcomes 2003;1:17.

27. Roos EM, Lohmander LS. The Knee injury and Osteoarthritis Outcome Score (KOOS): from joint injury to osteoarthritis. Health Qual Life Outcomes 2003;1:64.

28. Behrend H, Giesinger K, Giesinger JM, et al. The "forgotten joint" as the ultimate goal in joint arthroplasty: validation of a new patient-reported outcome measure. J Arthroplasty 2012;27:430-6.e1.

29. Giesinger JM, Kuster MS, Behrend H, et al. Association of psychological status and patient-reported physical outcome measures in joint arthroplasty: a lack of divergent validity. Health Qual Life Outcomes 2013;11:64.

30. Insall JN, Dorr LD, Scott RD, et al. Rationale of the Knee Society clinical rating system. Clin Orthop Relat Res 1989;248:13-14.

31. Ewald FC. The Knee Society total knee arthroplasty roentgenographic evaluation and scoring system. Clin Orthop Relat Res 1989;248:9-12.

32. Sells J. Summary of safety and effictiveness. Smith \& Nephew, Inc. LEGION cobalt chrome revision knee system, 2006. http://www. accessdata.fda.gov/cdrh_docs/pdf6/K060742.pdf

33. van Kempen RW, Schimmel JJP, van Hellemondt GG, et al. Reason for revision TKA predicts clinical outcome: prospective evaluation of 150 consecutive patients with 2-years followup. Clin Orthop Relat Res 2013;471:2296-302.

34. No authors listed. ICH Harmonised Tripartite Guideline. Statistical principles for clinical trials. International Conference on Harmonisation E9 Expert Working Group. Stat Med 1999;18:1905-42.

35. Blackwelder WC. "Proving the null hypothesis" in clinical trials Control Clin Trials 1982;3:345-53.

36. Julious SA. Sample sizes for clinical trials with normal data. Stat Med 2004;23:1921-86.

37. Lan KKG, DeMets DL. Discrete sequential boundaries for clinical trials. Biometrika. Biometrika 1093;70:659-63.

38. Westlake WJ. Symmetrical confidence intervals for bioequivalence trials. Biometrics 1976;32:741-4.

39. Verbeke G, Molenberghs $\mathrm{G}$. Linear mixed models for longitudinal data. New York: Springer, 2000.

40. No authors listed. The establishment and operation of clinical trial data monitoring committees for clinical trial sponsors 2016. http:// www.fda.gov/Regulatorylnformation/Guidances/ucm127069.htm 